$\mathbf{E}^{1}$ género Themeda Forssk. (Anthistiriinae, Sacchareae, Panicoideae, Poaceae) posee aproximadamente 18 especies distribuidas en los trópicos y subtrópicos del Viejo Mundo, principalmente Asia, África y Oceanía. Varias especies como T. quadrivalvis (L.) Kuntze, T. triandra Forssk. y T. villosa (Poir.) A. Camus han sido introducidas a otros países como especies exóticas. La primera es una maleza invasora nociva y las dos últimas son forrajeras $\mathrm{u}$ ornamentales (Clayton y Renvoize, 1986; Parsons y Cuthbertson, 2001).

La especie Themeda quadrivalvis (L.) Kuntze (Fig. 1) se considera nativa del sur de Asia, de la región de India y Pakistán. Actualmente, está distribuida en regiones tropicales y subtropicales del mundo (Keir y Vogler, 2006; Pandeya, 1967): desde China al este de Asia en Indonesia, Papúa Nueva Guinea y Australia, Medio Oriente, Madagascar; en América en cuatro estados de Estados Unidos; en Centroamérica está presente en Honduras y en varios países del Caribe; y en América del Sur en Argentina y Brasil (Catasus y Herrera, 1985; Keir y Vogler, 2006; Peterson et al. 2012; www.tropicos. org; USDA, 2012). Recibe el nombre común de "grader grass" o "habana oat grass" en Australia y Estados Unidos, o "yerba americana" en el Caribe (Peterson et al., 2012).

Esta nota relata el descubrimiento de Themeda quadrivalvis, la cual es maleza nociva y reglamentada en NOM-043-FITO-1999 de SAGARPA. Además de su naturaleza como maleza, el descubrimiento de este taxón, incrementa la diversidad de la familia Poaceae en México.

Antecedentes y descubrimiento. Existe un espécimen de Themeda quadrivalvis colectado en Sonora en 1884 por Edward Palmer. El espécimen está depositado en el herbario de ARIZ de la Universidad de Arizona. Su etiqueta carece de información de la localidad ya que sólo aparece el año de colecta y el colector. McVaugh (1956) escribió

\title{
ThemedA QUADRIVALVIS (SACCHAREAE, Panicoideae, Poaceae), una maleza REGLAMENTADA PRESENTE Y ESTABLECIDA EN el estado de Morelos, México
}

\author{
J. Gabriel Sánchez-Ken ${ }^{1,4}$, Rosa Cerros-Tlatilpa ${ }^{2}$ \\ Y HEIKE VIBRANS ${ }^{3}$
}

${ }^{1}$ Instituto de Biología, Universidad Nacional Autónoma de México, México, D.F., México

${ }^{2}$ Universidad Autónoma del Estado de Morelos, Cuernavaca, Morelos, México

${ }^{3}$ Colegio de Postgraduados, Montecillo, Estado de México, México

${ }^{4}$ Autor para la correspondencia: gabriels@ hotmail.co.uk

la biografía de Edward Palmer y de acuerdo a él, las colectas de Palmer en 1884 provienen de Sonora en lo que antiguamente se denominaba Colonia Lerdo, en la esquina noroeste del estado cerca de la frontera estatal con Baja California. Aparentemente, esta colecta fue fortuita y probablemente la especie no se estableció en Sono- ra, ya que no se volvió a encontrar. La especie no fue mencionada en el catálogo de las gramíneas de México (Dávila et al. 2006), ni en las gramíneas de Sonora (Beetle et al., 1991). Una situación similar ocurrió en Kansas cuando fue introducida al mezclarse con semillas para aves (Towne y Barnard, 2000) y tampoco logró es-

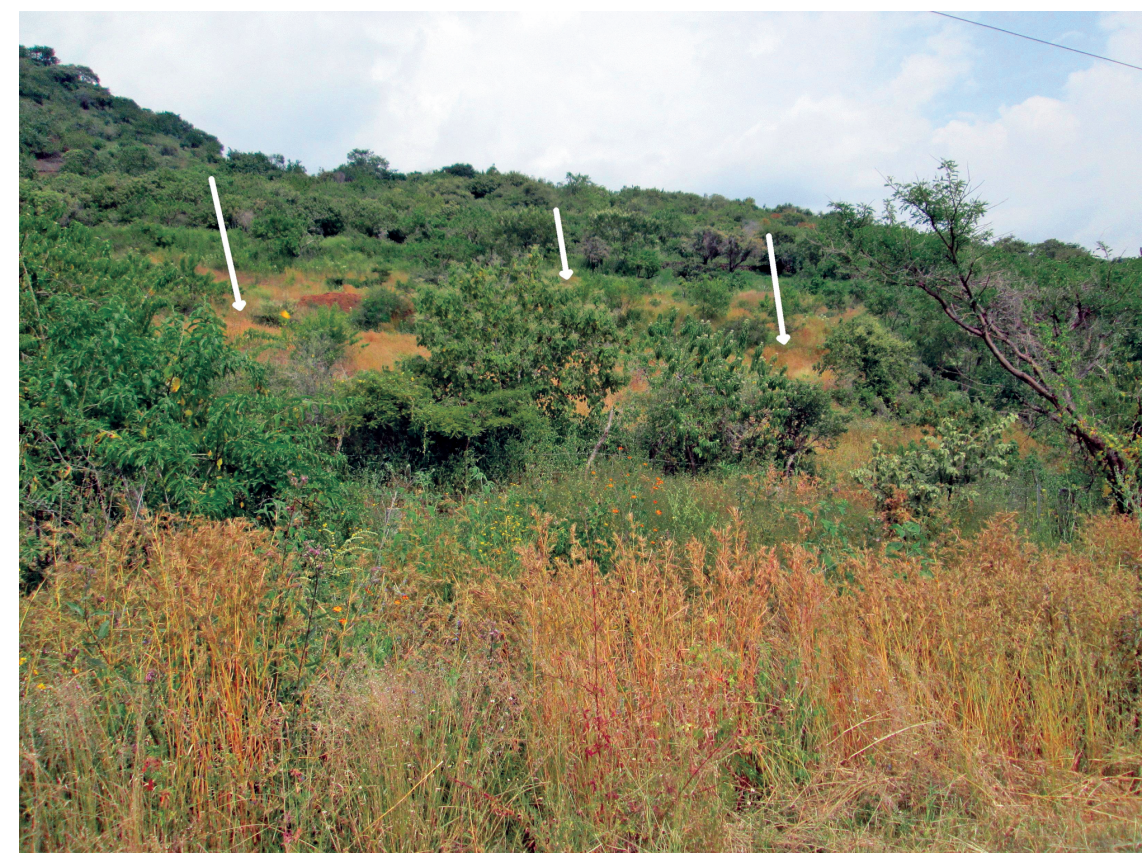

Figura 1. Población dominante de Themeda quadrivalvis en el estado de Morelos. 


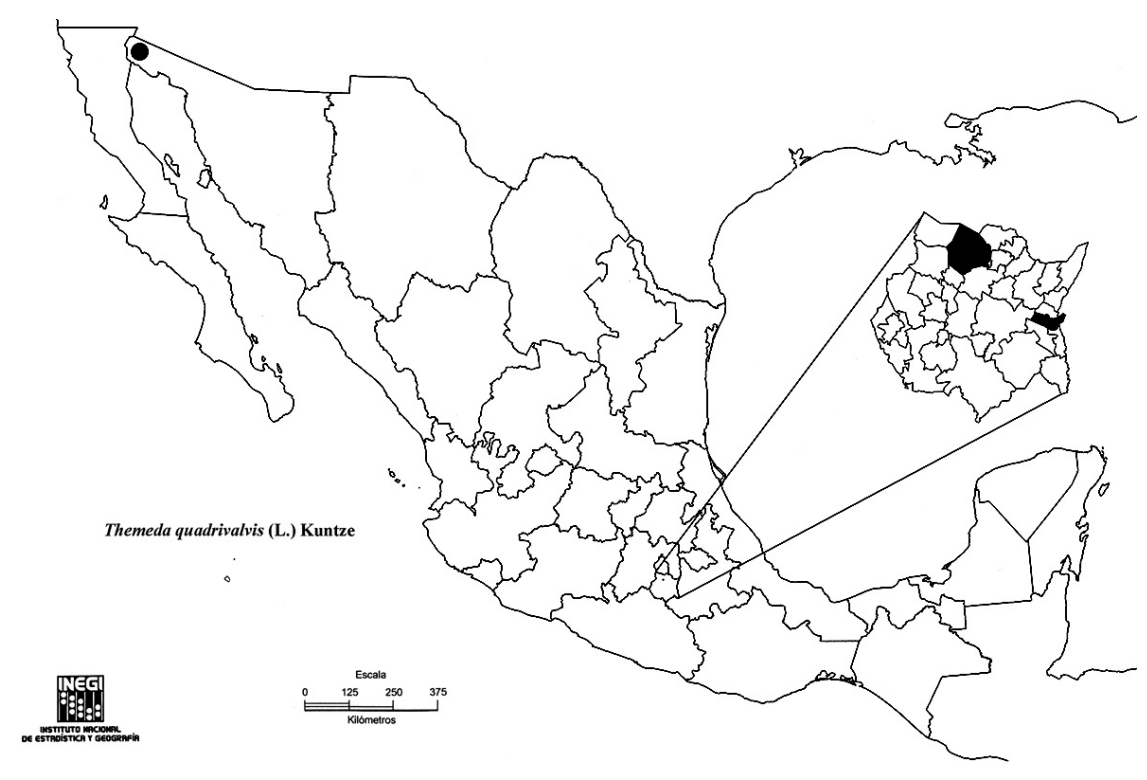

Figura 2. Distribución de Themeda quadrivalvis.

tablecerse. Desde entonces, la especie no había sido colectada en México y no existe otro ejemplar en ninguno de los principales herbarios del país. En 2006, la segunda autora colectó material de un pasto desconocido en la autopista entre Tepoztlán y Oacalco en el estado de Morelos. El material permaneció sin trabajar hasta 2012 cuando el primer autor revisó el material para el estado de Morelos, e identificó la especie como Themeda quadrivalvis.

En una inspección en el campo, en octubre y noviembre del 2012 se confirmó la existencia de poblaciones grandes de esta especie en el municipio de Tepoztlán. En el sitio de la primera colecta ahora cubre por lo menos una hectárea como especie dominante (Fig. 1 y 2). Además, está visible a los dos lados de la autopista durante varios kilómetros en dirección a Izúcar de Matamoros, por lo cual se encuentra bien establecida; de esta misma forma ocurrió en Louisiana, E.U., y Australia (Pandeya, 1967; Baird y Thieret, 1985; Keir y Vogler, 2006). El descubrimiento de la presencia Themeda quadrivalvis incrementa la diversidad de la familia Poaceae a nivel genérico y específico, tanto en México como en el estado de Morelos (Sánchez-Ken y Cerros-Tlatilpa, comm. pers.).
Características morfológicas. Las otras dos especies de Themeda que están presentes en América son Themeda arguens (L.) Hack. y T. triandra Forssk. Las tres especies son parecidas; sin embargo, son fácil de distinguir. Themeda quadrivalvis es una especie anual y en general tiene las espiguillas sésiles y pediceladas así como las aristas más cortas que las otras dos especies. El cuadro 1 muestra las principales diferencias entre las tres especies. Los especímenes colectados en Estados Unidos y Honduras varían de los colectados en Morelos en que las plantas son de menor tamaño y están ramificadas en la base de los culmos. Es probable que éstos especímenes crecieron en los márgenes de las poblaciones, pues se observó en las localidades de Morelos, que las plantas hacia los márgenes o aisladas de las poblaciones dominantes eran de menor talla que las del centro que eran más altas y carecían de ramificaciones.

Themeda quadrivalvis (L.) Kuntze, Revis. Gen. Pl. 763. 1891. Andropogon quadrivalvis L. in Murray, Syst. Veg., ed. 13. 758. 1774. Tipo: India (lectotipo: LINN-1211.5!, designado por Cafferty et al., Taxon 49(2): 246. 2000). Figura 3.
Themeda chinensis (A. Camus) S.L. Chen \& T.D. Zhuang, Bull. Bot. Res., Harbin 9(2): 59-60. 1989. Themeda ciliata (L. f.) Hack. subsp. chinensis A. Camus, Bull. Mus. Hist. Nat. (Paris) 26: 424. 1920. Themeda echinata Keng, Sinensia 10: 329. 1939. Basado en el mismo tipo. Tipo: China: Province Yunnan, 28 Nov 1906, J. Esquirol 1112 (isotipos: P!).

Themeda yuanmounensis S.L. Chen \& T.D. Zhuang, Bull. Bot. Res., Harbin 9(2): 60-61. 1989. Tipo: China: Province Yunnan, Yuanmou, 10 Sep 1963, J. Jiang 63-6833 (holotipo: JSBI; isotipos: PE, KUN, YUNU).

Plantas anuales, hasta $2 \mathrm{~m}$ de altura, formando carricillos, generalmente sin ramificaciones, erectas, a veces geniculadas en la base; entrenudos y nudos glabros, segundos nudos basales con raíces adventicias gruesas. Hojas con vainas glabras, aquilladas desde la base; lígulas 1-2.5 mm de largo, membranáceas; láminas hasta $60 \mathrm{~cm}$ de largo, 1-6 mm de ancho, lineares, planas a generalmente involutas, glabras en ambas superficies, con escasos pelos papilosos y setáceos en los márgenes hacia la base, márgenes escabrosos. Sinflorescencia hasta $130 \mathrm{~cm}$ de largo, muy compleja, formada por varios grupos de ramificaciones, cada grupo con una espata en cada nudo y parcialmente cubriendo la base, formada por varias ramas, con un profilo dorado a pardo en la base de cada rama, sobre el eje parten ramificaciones con una bráctea de la cual salen 5-10 ramérulos, cada uno de ellos con una espateóla formando una unidad, cada unidad con un profilo en su pedicelo; ramérulos formados por 2 pares de espiguillas homógamas y un triplete de espiguillas heterógamas; espiguillas homógamas insertadas más o menos al mismo nivel y formando un involucro al triplete de espiguillas heterógamas, 4-7 mm de largo, sin desarticulación, con tres brácteas, dorsiventralmente aplanadas; primera gluma tan larga como la espiguilla, multinervada con hasta 10 nervios, con escasos pelos pa- 


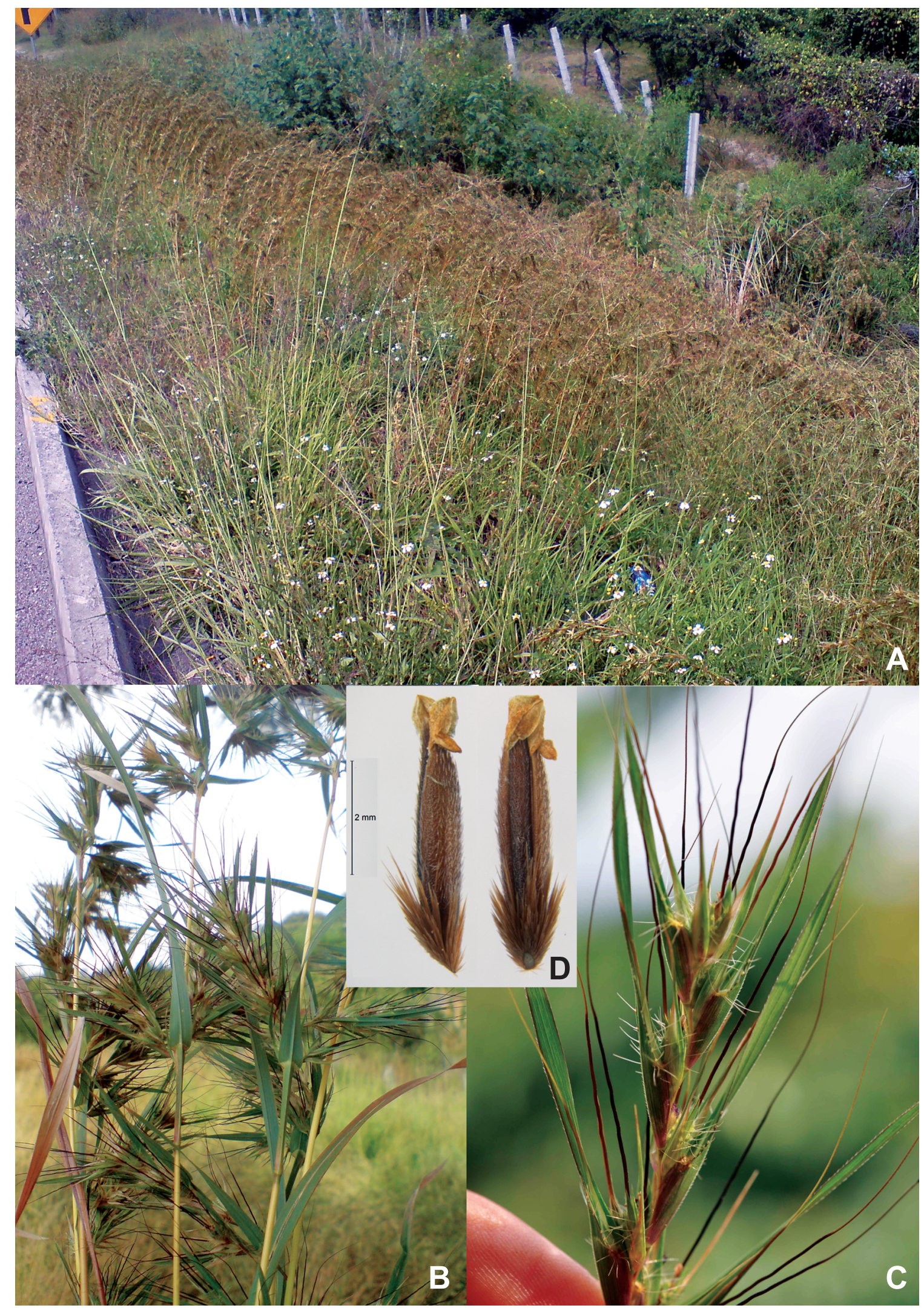

Figura 3. Themeda quadrivalvis. A. Población a un lado de la carretera. B. Acercamiento a la sinflorescencia. C. Acercamiento a los ramérulos. D. Espiguillas maduras. (Fotografías tomadas por los autores, excepto D por S. Guzmán). 
J. Gabriel SÁnchez-Ken ET AL.

Cuadro 1. Diferencias entre las especies del género Themeda presentes en América.

\begin{tabular}{|c|c|c|c|}
\hline & T. arguens & T. quadrivalvis & T. triandra \\
\hline Hábito & anual & anual & perenne \\
\hline $\begin{array}{l}\text { Pedicelo del grupo de } \\
\text { ramérulos, indumento }\end{array}$ & largo-piloso & glabro & glabro a corto piloso \\
\hline Espateóla indumento & $\begin{array}{l}\text { pelos papilosos } \\
\text { en toda la vaina }\end{array}$ & $\begin{array}{l}\text { pelos tuberculados } \\
\text { en el área ligular }\end{array}$ & $\begin{array}{l}\text { glabra, raro con } \\
\text { pelos papilosos }\end{array}$ \\
\hline $\begin{array}{l}\text { Espiguillas sésiles fértiles } \\
\text { (mm largo) }\end{array}$ & $9-11$ & $4-6$ & $6-14$ \\
\hline $\begin{array}{l}\text { Aristas de espiguillas } \\
\text { sésiles (cm largo) }\end{array}$ & $7-9$ & $4-5$ & $2.5-7$ \\
\hline $\begin{array}{l}\text { Callo del triplete de } \\
\text { espiguillas heterógamas } \\
\text { (mm largo) }\end{array}$ & $3-4$ & $(0.5) 1.5(3)$ & $2-4$ \\
\hline $\begin{array}{l}\text { Espiguillas homógamas } \\
\text { (mm largo) }\end{array}$ & $8-10$ & $4-7$ & $6-14$ \\
\hline $\begin{array}{l}1^{\text {a }} \text { gluma de espiguillas } \\
\text { homógamas indumento }\end{array}$ & glabra & $\begin{array}{c}\text { escasos pelos } \\
\text { papilosos setáceos }\end{array}$ & $\begin{array}{c}\text { glabra o papiloso } \\
\text { pilosa }\end{array}$ \\
\hline $\begin{array}{l}\text { Espiguillas pediceladas } \\
\text { (mm largo) }\end{array}$ & $8-10$ & $4.5-5.5$ & $6-14$ \\
\hline
\end{tabular}

pilosos setáceos hasta $3 \mathrm{~mm}$ de largo hacia arriba y hacia los márgenes, ápice redondo a agudo; segunda gluma casi tan larga como la espiguilla, ligeramente más corta que la primera gluma, 3-nervada; lema sólo una, hialina, sin nervaduras, glabra; lodículas 2; estambres 3 , cuando presentes rudimentarios; callo del triplete de espiguillas heterógamas, desarticulación oblicua, piloso, los pelos dorados a pardos; espiguillas heterógamas una sésil y dos pediceladas; espiguillas pediceladas, estériles, casi iguales, sobre un pedicelo de ca. $1.5 \mathrm{~mm}$ de largo, aplanadas, glabras; espiguillas sésiles fértiles, 4-6 $\mathrm{mm}$ de largo excluyendo la arista de la lema fértil, terete, pardo obscuras cuando maduras; primera gluma tan larga como la espiguilla, encerrando a la segunda gluma, coriácea, pajiza a pardo obscura cuando madura, 10nervada, las nervaduras inconspicuas, esparcidamente estrigosas, ápice truncado; segunda gluma tan larga como la espiguilla, coriácea, estramínea a pardo obscura cuando madura, la parte expuesta convexa y estrigosa, 3-nervada, las nervaduras laterales aquilladas hacia adentro, los márgenes glabros y cubiertos por la segunda gluma; lema inferior estéril, hialina, más corta que las glumas; lema fértil reducida a una arista $4-5 \mathrm{~cm}$ de largo, la parte basal blanquecina y después pardo obscuro hacia arriba, retorcida, flexuosa, retrorsamente hirsutela; pálea fértil menos de $1 \mathrm{~mm}$ de largo, hialina; lodículas 2, ca. $1 \mathrm{~mm}$ de largo, oblongas, truncadas, con dos lóbulos en cada esquina; estambres 3 , anteras 1.4-1.5 $\mathrm{mm}$ de largo, amarillas; cariópside 3-3.1 mm de largo, ca. $0.6 \mathrm{~mm}$ de ancho, obovada, estramínea, embrión casi la mitad del largo de la cariópside, hilo 1/6 del largo de la cariópside, oblongo, pardo obscuro.

Especímenes revisados: México. MORELOS: Mpio. Tepoztlán, Autopista Tepoztlán-Caseta de Oacalco, vegetación secundaria, $1,560 \mathrm{~m}, 28$ sep 2006, R. Cerros T., A. Flores et al. 2675 (HUMO, MEXU); Autopista Tepoztlán a Oacalco, a los lados de la carretera, varias poblaciones, plantas expuestas más pequeñas, $18^{\circ} 58^{\prime}$ 08.7" N, 99 04' 18.3 ", 1590 m, 20 oct 2012, J.G. Sánchez-Ken, H. Vi- brans \& E. García M. s.n. (MEXU, IBUG, XAL). SONORA: Sin localidad, in 1884, E. Palmer s.n. (ARIZ!).

Biología. Pandeya (1967) estudió la biología y ecología de la especie en la India, mientras que Keir y Vogler (2006) hicieron una revisión detallada de lo que se conoce hasta ahora de Themeda quadrivalvis en Australia, describieron la biología, germinación, dispersión, su amenaza a la biodiversidad, su cultivo y valor como forraje, su declaración como maleza en Australia así como los métodos de control mecánico y químico de la especie. En Australia se distribuye entre la latitud $17^{\circ}$ y $33^{\circ} \mathrm{S}$. Está adaptada a una amplia gama de tipos climáticos ya que se ha registrado de regiones con precipitaciones promedios anuales entre 375 hasta más de $3000 \mathrm{~mm}$ anuales. Prefiere suelos bien drenados con textura mediana, arenas limosas a limos y prospera menos en sitios anegados, como son suelos arenosos o arcillas. Es una especie dominante en pastizales naturales en la India. Las plantas empiezan a crecer en junio, después de la temporada de lluvias y empiezan a florecer en septiembre. Para octubre las plantas ya producen semillas (Pandeya, 1967). Al considerar las observaciones de campo, parece que en México sigue un ciclo similar.

La especie tiene la capacidad de formar monocultivos que producen una buena cantidad de heno para pastura, aunque con una baja palatabilidad, sobre todo después de la floración (Pandeya, 1967; Keir y Vogler, 2006). Esta producción de biomasa también la hace propicia para incendios de temporada seca (Keir y Vogler, 2006).

Pandeya (1967) reportó la producción de 1332 cariópsides en promedio por planta, con un mínimo de 624, aunque en las plantas presentes en Estados Unidos, México y Honduras el número de cariópsides parece ser mucho menor. Puede producir semillas sin polinización mediante de la apomixis facultativa. Los frutos tienen una baja latencia. Además, no son muy longe- 
vos en el suelo, y no germinan bajo sombra fuerte (Keir \& Vogler, 2006). Las diásporas tienen una arista higroscópica y móvil que ayuda a la semilla enterrarse al suelo. Aparentemente, las cariópsides maduras germinan con una mortalidad muy baja de plántulas (Pandeya, 1967).

La diásporas se dispersan a través de la exozoocoría con animales y humanos, ya que de manera natural tiene un alcance de dispersión muy corta por el peso de las espiguillas (Bishop, 1981; Parsons y Cuthbertson, 2001). En Australia, fue introducida accidentalmente a nuevas regiones repetidamente con pacas comerciales de pasto seco para forraje (Keir \& Vogler, 2006), y en otras regiones con alimento para aves. Para México se desconoce la vía de introducción.

Impacto y reglamentación. La especie invade pastizales, tanto naturales como potreros, cultivos anuales, caña de azúcar, plantaciones de cultivos tropicales perennes, orillas de vías y bosques tropicales abiertos (Keir y Vogler, 2006). La capacidad de formar poblaciones vigorosas y puras, baja palatabilidad, número de cariópsides producidas por planta, alta tasa de germinación, mortalidad baja de plántulas, la capacidad de reproducirse sexualmente y asexualmente mediante la apomixis facultativa y su tetraploidía son características que confieren a la especie un elevado nivel de peligrosidad como maleza (Pandeya, 1967; Birari, 1980; Keir y Vogler, 2006). Estas características de la planta, junto con su susceptibilidad a incendios, pueden reducir la biodiversidad local en forma irreversible y disminuir la productividad pecuaria y agrícola (Keir y Vogler, 2006; Vogler y Owen, 2008).

Themeda quadrivalvis es una maleza nociva invasora en los países donde está presente como exótica o introducida. En Australia fue introducida en los años 1930 y se expandió rápidamente, en la actualidad es una maleza nociva importante (Keir y Vogler, 2006). México y otros países que tienen hábi- tats y climas similares a los australianos han declarado a ésta especie como maleza nociva cuarentenaria o reglamentaria. En México la especie está incluida en la NOM-043-FITO-1999 como especie cuarentenaria y requiere acciones de erradicación en el caso de ser encontrada en otras localidades o regiones del país.

Control. Existen varias formas de control de la maleza en los diferentes estados del ciclo de vida de las plantas. Puede ser controlada antes de la germinación, en la etapa de plántulas, antes de la floración y antes de la maduración de las cariópsides. Las formas de control existentes son mecánicas y químicas. Debido a que se trata de una planta anual, las medidas deben ser dirigidas principalmente a evitar la formación de nuevas semillas. Los métodos mecánicos incluyen el corte antes de o durante la floración combinado con descansos durante varias estaciones de lluvia. Si ya existen inflorescencias, deben ser quemadas. Los métodos químicos por su naturaleza no deseados, incluyen el uso de glifosfato, paraquat, y otros más con sus debidos cuidados (Keir y Vogler, 2006).

Recomendaciones. El descubrimiento de Themeda quadrivalvis obliga a establecer acciones de control o erradicación de la maleza, para evitar que se extienda en otros estados de la República Mexicana. El Servicio Nacional de Sanidad, Inocuidad y Calidad Agroalimentaria (SENASICA), de la Secretaría de Agricultura, Ganadería, Desarrollo Rural, Pesca y Alimentación (SAGARPA) es el organismo de gobierno federal encargado del manejo, control y erradicación de malezas que conforman la lista de la NOM043-FITO-1999. SENASICA ha implementado una campaña de detección y erradicación de focos de infestación de malezas reglamentadas en varios estados del país (http://www.senasica. gob.mx/?id=4522). Con la notificación a SENASICA de la presencia de ésta maleza en el estado de Morelos se tendrá la oportunidad de desarrollar un plan de manejo de Themeda quadrivalvis en el próximo verano.

\section{Agradecimientos}

Agradecemos a la Biól. Susana Guzmán de la Unidad de Informática para la Biodiversidad, UNIBIO del Instituto de Biología, UNAM, la toma de la fotografía de las espiguillas.

\section{Literatura citada}

Baird J.R. y Thieret J.W. 1985. Notes on Themeda quadrivalvis (Poaceae) in Lousiana. Isleya 2:129-137.

Birari S.P. 1980. Apomixis and sexuality in Themeda Forssk. at different ploidy levels (Gramineae). Genetica 54:133-139.

Bishop H.G. 1981. Grader grass - a nuisance weed. Queensland Agricultural Journal 107:235-239.

Beetle, A.A., Gordon D.J., Navarro-Córdova A. y Alcaráz-Flores A. 1991. Gramíneas de Sonora. Secretaría de Agricultura y Recursos Hidráulicos, México, DF. 174 pp.

Catasús L. y Herrera P. 1985. Revisión del género Themeda (Poaceae) en Cuba. Acta Botánica Cubana 35:1-3.

Clayton W.D. y Renvoize S.A. 1986. Genera Graminum, Grasses of the World. The Board of Trustees of the Royal Botanic Gardens, Kew.

Dávila P., Mejía-Saulés M.T, Gómez-Sánchez M., Valdés-Reyna J., Ortíz J.J., Morín C., Castrejón J. y Ocampo A. 2006. Catálogo de las Gramíneas de México. Universidad Nacional Autónoma de México-Comisión Nacional para el Conocimiento y Uso de la Biodiversidad, México, D.F.

Keir A.F. y Vogler W.D. 2006. A review of current knowledge of the weedy species Themeda quadrivalvis (grader grass). Tropical Grasslands 40:193-201.

McVaugh, R. 1956. Edward Palmer, plant explorer of the American West. University of Oklahoma Press, Norman.

Pandeya S.C. 1967. Some observations on the autoecology of Themeda quadrivalvis (Linn.) O. Ktze. var. quadrivalvis. Proceedings: Plant Sciences 65:63-75.

Parsons W.T. y Cuthberston E.G. 2001. Noxious Weeds of Australia. 2nd ed. CIRO Publishing, Collinwood. 


\section{J. Gabriel SÁnchez-Ken ET AL.}

Peterson P.M., Soreng R.J. y Smith S.F. 2012. Poaceae. En: Acevedo-Rodríguez P. y Strong M.T. eds. Catalogue of Seed Plants of the West Indies, pp. 714-766, Smithsonian Contributions to Botany 98. Smithsonian Institution Scholarly Press, Washington, D.C.

SENASICA. Servicio Nacional de Sanidad, Inocuidad y Calidad Agroalimentaria, SAGARPA, Secretaría de Agricultura,
Ganadería, Desarrollo Rural, Pesca y Alimentación, México. [http://www. senasica.gob.mx/?id=4522]

Towne E.G. y Barnard I. 2000. Themeda quadrivalvis (Poaceae: Andropogoneae) in Kansas: An exotic plant introduced from birdseed. Sida 19:201-203.

United States Department of Agriculture. USDA. 2012. Natural Resources Conservation Service, Plants Data- base. [http://plants.usda.gov/java/profile? symbol=THQU] (Accesado Nov. 2012)

Vogler W.D. y Owen N.A. 2008. Grader grass (Themeda quadrivalvis): changing savannah ecosystems. En: van Klinken R.D., Osten V.A., Panetta F.D. y Scanlan J.C. Eds. Sixteenth Australian Weeds Conference, pp. 213-214, Queensland Weeds Society Cairns.

Recibido: 28 de noviembre de 2012

Aceptado: 21 de febrero de 2013 